\section{Eficacia del paracetamol intravenoso para el cierre del conducto arterioso en recién nacidos prematuros}

\section{RESUMEN}

Antecedentes: los inhibidores de la ciclooxigenasa, como la indometacina y el ibuprofeno, que se utilizan para el cierre del conducto arterioso tienen efectos adversos significativos en el recién nacido, por lo que es importante explorar nuevas alternativas de tratamiento eficaces y seguras. Una de ellas podría ser el paracetamol intravenoso.

Objetivo: evaluar la eficacia y seguridad del paracetamol intravenoso para el cierre del conducto arterioso en recién nacidos prematuros.

Material y métodos: se realizó un estudio prospectivo, experimental, en recién nacidos prematuros con diagnóstico ecocardiográfico de persistencia de conducto arterioso hemodinámicamente significativo; se les administró paracetamol intravenoso (15 mg/kg/do) cada seis horas por tres a siete días. En la evolución de estos pacientes se trató de identificar el cierre del conducto arterioso y los efectos adversos del paracetamol.

Resultados: se incluyeron nueve recién nacidos prematuros con edad gestacional de 33.2 semanas y peso medio de 1509 gramos. Cinco (55\%) tuvieron índice de Yeh $\geq 3$, por ecocardiografía el diámetro interno medio del conducto arterioso fue de $2.81 \pm 1.05 \mathrm{~mm}$ con una relación aurícula izquierda/raíz aortica media (Al/Ao) de $1.5 \pm 0.32$. Se detectó el cierre del conducto arterioso en ocho (89\%) pacientes; el tiempo medio de cierre fue de $3.4 \pm 1.7$ días. No se identificaron reacciones adversas al paracetamol.

Conclusiones: el paracetamol intravenoso puede ser una alternativa de tratamiento eficaz y segura para el cierre del conducto arterioso hemodinámicamente significativo; sin embargo, se requieren más estudios para validar estas observaciones iniciales.

Palabras clave: conducto arterioso, paracetamol, prematuro.

\section{Efficacy of intravenous paracetamol for closure of patent ductus arteriousus in preterm newborns}

\section{ABSTRACT}

Background: Ciclooxigenase inhibitors such as indometacin and ibuprofen, currently used for closure of the ductus arteriousus (DA) are associated with significant adverse effects on the newborn, so it is
Henry Sergio Carrillo-Arteaga ${ }^{1}$ Jessica Valencia-Avendaño Lucía Oliveros-Ruiz ${ }^{3}$

${ }^{1}$ Neonatólogo adscrito al Servicio de Neonatología. Hospital de Especialidades de la Ciudad de México Dr. Belisario Domínguez. Secretaría de Salud del Distrito Federal. Profesor Adjunto de Neonatología, Universidad Nacional Autónoma de México.

${ }^{2}$ Residente de Neonatología del Hospital de Especialidades de la Ciudad de México Dr. Belisario Domínguez. Secretaría de Salud del Distrito FederalUniversidad Nacional Autónoma de México.

${ }^{3}$ Médico Cardiólogo del Hospital de Especialidades de la Ciudad de México Dr. Belisario Domínguez. Secretaría de Salud del Distrito Federal.

Recibido: 8 de abril del 2014

Aceptado: 10 de diciembre del 2014

Correspondencia: Dr. Henry Sergio Carrillo Arteaga

Unidad de Cuidados Intensivos Neonatales

Hospital de Especialidades de la Ciudad de México Dr. Belisario Domínguez

Avenida Tláhuac 4866

CP 09790, México, D.F.

Teléfono: 58500026

sercarrillo@yahoo.com

Este artículo debe citarse como

Carrillo-Arteaga HS, Valencia-Avendaño J, OliverosRuiz L. Eficacia del paracetamol intravenoso para el cierre del conducto arterioso en recién nacidos prematuros. Acta Pediatr Mex 2015;36:18-25. 
important to explore new alternatives of effective and safe treatment. One of these could be intravenous paracetamol.

Objective: Evaluate the efficacy and safety of intravenous paracetamol for the closure of DA in preterm newborns.

Material and Methods: We performed a prospective, experimental study, in preterm newborns with echocardiographic diagnosis of hemodynamically persistent ductus arteriousus (PDA), who were administered intravenous paracetamol $15 \mathrm{mg} / \mathrm{kg} /$ day every six hours for three to seven days. In the follow up of these patients we looked for DA closure and side effects of paracetamol.

Results: We included nine preterm newborn with gestacional age and weight mean of 33.2 weeks and 1509 grams respectively. Five patient $(55 \%)$ had Yeh index $\geq 3$ and by echocardiography the mean internal diameter of the DA was $2.81 \pm 1.05 \mathrm{~mm}$., with a ratio of left atrium/ aortic root $(\mathrm{LA} / \mathrm{Ao}): 1.5 \pm 0.32$. Closure of DA was detected in eight (89\%) patients; closure average time was $3.4 \pm 1.7$ days. No adverse reactions were detected.

Conclusions: Intravenous paracetamol can be an effective and safe alternative treatment for closure of hemodinamically significant DA; however further studies are needed to validate these initial observations.

Key words: Ductus arteriousus, paracetamol, preterm.

\section{INTRODUCCIÓN}

La persistencia del conducto arterioso ocurre en 7 a $25 \%$ de los recién nacidos prematuros y está asociada con incremento considerable de la mortalidad neonatal. ${ }^{1,2}$ En los recién nacidos prematuros las concentraciones bajas de oxígeno en el útero y las concentraciones elevadas de prostaglandinas contribuyen a mantener la persistencia del conducto arterioso. ${ }^{3,4}$ La persistencia del conducto arterioso hemodinámicamente significativo se puede identificar por criterios clínicos que se agrupan en el índice de Yeh, que incluye la determinación de taquicardia, pulsos amplios, características del soplo cardíaco, precordio hiperdinámico e índice cardiotorácico, con asignación de una puntuación de 0 a 2 de acuerdo con la gravedad de cada parámetro. ${ }^{5,6}$ Un índice de Yeh $\geq 3$ es sugestivo de persistencia del conducto arterioso hemodinámicamente significativo. ${ }^{3,5,7}$ En el ecocardiograma el diámetro interno $\geq 1.6 \mathrm{~mm}$, con una relación aurícula izquierda/raíz aortica igual o mayor a 1.2 sugieren persistencia significativa del conducto arterioso..$^{8,9} \mathrm{El}$ diagnóstico de persistencia del conducto arterioso en el prematuro debe ser ecocardiográfico ya que los signos clínicos son con frecuencia poco fiables y puede existir un conducto arterioso grande con gran paso de flujo de izquierda a derecha en ausencia de síntomas. ${ }^{10} \mathrm{El}$ tratamiento farmacológico actual para el cierre del conducto arterioso es la administración de indometacina o ibuprofeno, que promueven la constricción del conducto arterioso por bloqueo de la síntesis de prostaglandinas por inhibición del segmento ciclooxigenasa de la 
prostaglandina sintetasa; ;1,12 sin embargo, estos fármacos tienen efectos adversos como hemorragia, plaquetopenia, enterocolitis, insuficiencia renal e hiperbilirrubinemia. ${ }^{13,14,15} \mathrm{~A}$ diferencia de éstos, el paracetamol parece inhibir el segmento peroxidasa de la prostaglandina sintetasa. La peroxidasa es activada a concentraciones de peróxido diez veces más bajas en relación a la ciclooxigenasa y su inhibición mediada por el paracetamol es facilitada por la reducción de las concentraciones locales de peróxido en situaciones como hipoxia. Esto permite que la inhibición de la peroxidasa sea efectiva en condiciones en que la inhibición de la ciclooxigenasa es menos activa. ${ }^{16,17}$ Estas observaciones estimularon en los autores el interés por responder a la siguiente interrogante: ¿cuál es la eficacia y seguridad del paracetamol intravenoso para el cierre del conducto arterioso hemodinámicamente significativo?, planteándonos como objetivo evaluar estos parámetros. Nuestra intención fue confirmar que el paracetamol es eficaz y seguro para el cierre del conducto arterioso.

\section{MATERIAL Y MÉTODOS}

Estudio prospectivo, experimental, en el que se incluyó a recién nacidos menores de 37 semanas de gestación, ingresados en la Unidad de Cuidados Intensivos Neonatales del Hospital de Especialidades de la Ciudad de México Dr. Belisario Domínguez, entre enero y junio de 2013, con diagnóstico de persistencia del conducto arterioso hemodinámicamente significativo, determinado por ecocardiografía con los siguientes parámetros: diámetro interno $\geq 1.6 \mathrm{~mm}$ y relación aurícula izquierda/raíz aortica $\geq 1.2$. Se excluyeron los pacientes con cardiopatías complejas, además de persistencia del conducto arterioso hemodinámicamente no significativo, enterocolitis necrosante IIB o mayor, hemorragia intraventricular mayor al grado II, insuficiencia renal aguda, trombocitopenia menor a 20 000/ $\mathrm{mm}^{3}$. Se eliminaron del estudio los pacientes que fueron trasladados a otra unidad hospitalaria en forma definitiva o que presentaron reacciones adversas al paracetamol. Invariablemente se solicitó consentimiento informado a los padres o al tutor para incluir al paciente en el el estudio.

A los recién nacidos prematuros con los criterios de ingreso al estudio se solicitó que se les practicara ecocardiografía inicial al tercer día de vida o cuando se identificaran signos clínicos de persistencia del conducto arterioso durante su estancia hospitalaria. Al corroborar la persistencia del conducto arterioso se solicitaron estudios de laboratorio basales (antes de iniciar tratamiento con paracetamol): biometría hemática, electrólitos séricos, pruebas de función renal, pruebas de función hepática, tiempos de coagulación, hemocultivo y ultrasonido transfontanelar. En los pacientes seleccionados se indicó restricción hídrica intravenosa de 80 a 120 mL/kg/día entre el tercer y séptimo días de vida; después de los siete días la terapia intravenosa fue restringida a $130 \mathrm{~mL} / \mathrm{kg} /$ día. Los pacientes recibieron furosemida intravenosa sólo después del quinto día de vida ( $1 \mathrm{mg} / \mathrm{kg} /$ do cada 12 horas) si no recibían líquidos vía oral. Cuando el aporte por vía oral fue mayor de $100 \mathrm{~mL} / \mathrm{kg} /$ día se prescribió hidroclorotiazida (1 mg/kg/do cada 12 horas) y espironolactona (1 mg/kg/do cada 24 horas).

Una vez establecido el diagnóstico ecocardiográfico de persistencia del conducto arterioso hemodinámicamente significativo, se administró paracetamol intravenoso (Bristol-Myers Squibb $^{\circledR}$, frasco ámpula de $100 \mathrm{~mL}$ con $1 \mathrm{~g}$ de paracetamol) en dosis de $15 \mathrm{mg} / \mathrm{kg} /$ do cada seis horas durante tres a siete días. Todos los pacientes recibieron tratamiento convencional acorde con las afecciones acompañantes; se incluyeron administración de surfactante, antibióticos, glucosa, soluciones parenterales, nutrición parenteral y las diferentes modalidades de oxigenoterapia: oxígeno indirecto, presión positiva continua en la vía aérea nasal o venti- 
lación mecánica. Se registraron datos generales: peso, edad gestacional y enfermedades acompañantes: síndrome de dificultad respiratoria por déficit de surfactante, neumonía y sepsis temprana. Se registró la edad de aparición de los signos de persistencia del conducto arterioso sintomático, índice de Yeh, edad al realizar el ecocardiograma, signos ecocardiográficos de conducto arterioso significativo, edad de inicio de paracetamol, identificación de cierre o falta de cierre del conducto arterioso, tiempo de cierre y reacciones adversas debidas a la administración de paracetamol: hemorragia intraventricular diagnosticada por ultrasonido transfontanelar, insuficiencia renal detectada por elevación de creatinina sérica y del nitrógeno ureico e insuficiencia hepática: elevación de transaminasa glutámico oxalacética y transaminasa glutámico pirúvica, bilirrubina directa, fosfatasa alcalina, gammaglutamil transpeptidasa y descenso de albumina. Cuando el cierre del conducto arterioso se produjo al tercer día de tratamiento se suspendió la administración de paracetamol. Si no se lograba el cierre del conducto arterioso en este tiempo el tratamiento se continuó hasta siete días y, al octavo día, se solicitaron nuevos exámenes de laboratorio, ecocardiografía y ultrasonido transfontanelar. Si en el control ecocardiográfico se identificaba cierre del conducto arterioso el tratamiento era suspendido al concluir el séptimo día; si no se conseguía cierre del conducto arterioso después de este tiempo el paciente era operado. El análisis estadístico se hizo utilizando frecuencias para las condiciones generales y determinación de media y desviación estándar. Se utilizó el programa estadístico SPSS versión 20.

\section{RESULTADOS}

Entre enero y junio de 2013 ingresaron a la Unidad de Cuidados Intensivos Neonatales del Hospital de Especialidades de la Ciudad de México Dr. Belisario Domínguez 67 recién nacidos prematuros (52\% del total de los ingresos) y se diagnosticó persistencia del conducto arterioso por ecocardiografía en 12 (18\%) pacientes. Se incluyeron nueve pacientes y se excluyeron tres: uno por cardiopatía congénita compleja y dos por persistencia del conducto arterioso hemodinámicamente no significativo. Ningún paciente fue trasladado a otra unidad hospitalaria. Seis $(67 \%)$ pacientes eran del sexo masculino y tres (33\%) del femenino. La edad gestacional media fue de 33 semanas (rango: 30-36); peso promedio de 1509 gramos con intervalos de 1002 a 2294 gramos. Los diagnósticos más frecuentes asociados a la persistencia del conducto arterioso fueron: síndrome de dificultad respiratoria por déficit de surfactante en cuatro (44\%) pacientes, neumonía en 8 pacientes (89\%), enterocolitis necrosante en $2(22 \%)$ pacientes y sepsis neonatal temprana en nueve (100\%) pacientes.

En seis pacientes $(67 \%)$ se identificó soplo sistólico y en dos (22.2\%) soplo continuo; en 1 paciente $(11 \%)$ no se logró identificar este signo. El soplo cardíaco fue identificado a una edad media de $7 \pm 1.2$ días. Presentaron taquicardia cinco (57\%) y diferencial de pulso amplia (mayor a $20 \mathrm{mmHg}$ ) siete $(78 \%)$ pacientes. La edad media de identificación de taquicardia fue de $7 \pm 1.5$ días y de la diferencial de pulso amplia de $2 \pm 1.2$ días. Se identificó hiperactividad precordial en seis $(67 \%)$ pacientes, con una edad media de identificación de $6 \pm 1.2$ días. El índice cardiotorácico fue mayor a 0.6 en dos (22\%) pacientes. Cinco (55\%) pacientes tuvieron índice de Yeh mayor a 3. Ocho (89\%) pacientes requirieron ventilación mecánica, cuatro $(44 \%)$ ventilación mecánica más presión positiva continua en las vías respiratorias y nueve $(100 \%)$ oxígeno por cámara cefálica. El tiempo medio de requerimiento de $\mathrm{O}_{2}$ suplementario fue de 18 días.

La edad media en el estudio ecocardiográfico fue de 6 días. Respecto al informe de ecocardiografía el diámetro interno medio del conducto arterioso 
fue de $2.81 \pm 1.05 \mathrm{~mm}$; la longitud media de $3.38 \pm 1.45 \mathrm{~mm}$ y el gradiente transductal de $31.1 \pm 9.54 \mathrm{mmHg}$, con una relación aurícula izquierda/raíz aortica media de $1.5 \pm 0.32$.

La edad media de inicio de tratamiento fue de seis días. Se logró cierre del conducto arterioso hemodinámicamente significativo, corroborado por ecocardiografía, en ocho (89\%) pacientes. El tiempo medio de cierre fue de $3.4 \pm 1.7$ días. No se logró cierre del conducto arterioso en un paciente $(11 \%)$ que había recibido manejo previo con ibuprofeno oral por tres días y que finalmente requirió tratamiento quirúrgico. El Cuadro 1 muestra el seguimiento de los pacientes tratados con paracetamol.

Las concentraciones de enzimas hepáticas, las pruebas de función renal y el ultrasonido transfontanelar pre- y postratamiento fueron normales en todos los pacientes. Un paciente tuvo plaquetopenia debido a sepsis por Klebsiella; mejoró con tratamiento antibiótico específico.

\section{Análisis}

En este estudio identificamos persistencia del conducto arterioso en $18 \%$ de los 67 pacientes, lo que concuerda con lo descrito en la literatura especializada..$^{3,9,16,17}$ En nueve (75\%) de éstos se identificó por ecocardiografía persistencia del conducto arterioso hemodinámicamente significativo. De éstos $44 \%$ pesaban menos de 1500 gramos. La edad gestacional media de 33 semanas y el peso medio de 1500 gramos señalan que la afección fue identificada con más frecuencia en el grupo de pacientes de 1000 a 1500 gramos, lo que seguramente se debe a que el porcentaje mayor de prematuros que ingresó a nuestra Unidad de Cuidados Intensivos Neonatales en el período de estudio pesaba más de 1000 gramos. La mayoría de estos pacientes tenía neumonía (89\%) y sepsis temprana (100\%), lo que se relaciona con antecedentes infecciosos de la madre y no necesariamente con datos de respuesta inflamatoria y cultivos positivos ya que sólo en uno de estos pacientes se identificó hemocultivo positivo para Klebsiella. Sólo en $44 \%$ de los pacientes se identificó síndrome de dificultad respiratoria por déficit de surfactante, lo que se debe a que la mayoría de ellos no fueron prematuros extremos. El hecho de que $89 \%$ de los pacientes haya requerido ventilación mecánica puede deberse a la magnitud del cortocircuito transductal como manifestación inicial de persistencia del conducto arterioso significativo, aunque es posible también que el requerimiento incrementado de oxígeno suple-

Cuadro 1. Seguimiento de pacientes tratados con paracetamol

\begin{tabular}{ccccccccc}
\hline Núm. & $\begin{array}{c}\text { EG } \\
\text { (semanas) }\end{array}$ & $\begin{array}{c}\text { Peso } \\
(\mathbf{g})\end{array}$ & $\begin{array}{c}\text { DI CA } \\
(\mathbf{m m})\end{array}$ & Al/Ao & $\begin{array}{c}\text { Edad inicio con } \\
\text { paracetamol (días) }\end{array}$ & $\begin{array}{c}\text { Tiempo con } \\
\text { paracetamol } \\
\text { (días) }\end{array}$ & $\begin{array}{c}\text { Cierre por } \\
\text { ecocardiografía }\end{array}$ & $\begin{array}{c}\text { Reacciones } \\
\text { adversas }\end{array}$ \\
\hline 1 & 30 & 1218 & 3 & 2.0 & 10 & 3 & Sí & No \\
2 & 33 & 1470 & 2 & 1.2 & 5 & 3 & Sí & No \\
3 & 33 & 1512 & 5 & 2.0 & 7 & 7 & No & No \\
4 & 33 & 1602 & 3 & 1.5 & 3 & 3 & Sí & No \\
5 & 35 & 1410 & 3 & 1.8 & 3 & 3 & Sí & No \\
6 & 36 & 2294 & 4 & 1.2 & 2 & 3 & Sí & No \\
7 & 33 & 1360 & 2 & 1.3 & 19 & 3 & Sí & No \\
8 & 36 & 1715 & 4 & 1.5 & 3 & 3 & Sí & No \\
9 & 30 & 1002 & 2 & 1.4 & 3 & 7 & 3
\end{tabular}

EG: edad gestacional en semanas; DI CA: diámetro interno del conducto arterioso. Al/Ao: relación aurícula izquierda/raíz aórtica. 
mentario sea debido a las condiciones clínicas acompañantes como sepsis y neumonía. ${ }^{10,18}$

Los signos clínicos más frecuentes fueron el soplo cardíaco y la presión diferencial de pulso amplia. A excepción de la diferencial de pulso amplia los otros signos de persistencia del conducto arterioso significativo se identificaron en promedio a la semana de vida. Estos datos reafirman la importancia de realizar el diagnóstico ecocardiográfico temprano de persistencia del conducto arterioso en todos los recién nacidos prematuros, ya que los signos clínicos típicos de persistencia del conducto arterioso no siempre son evidentes inicialmente; pueden presentarse tardíamente como se reporta en la literatura especializada: los criterios ecocardiográficos de un cortocircuito izquierda-derecha significativo habitualmente preceden a los síntomas clínicos por un intervalo de $2-3$ días, ${ }^{19}$ de modo que si se toman en cuenta sólo estos signos clínicos el diagnóstico podría retrasarse y el recién nacido prematuro estaría expuesto a una mayor frecuencia de complicaciones propias de la persistencia del conducto arterioso. ${ }^{19,20}$

En nuestro estudio los hallazgos ecocardiográficos no tuvieron una correlación clínica adecuada ya que, si bien en la mayoría de los pacientes se identificó persistencia del conducto arterioso significativo por ecocardiografía, sólo en poco más de $50 \%$ de los pacientes el índice de Yeh fue mayor o igual a 3. Esta discordancia entre los hallazgos ecocardiográficos y clínicos se debe a que en el recién nacido prematuro las resistencias vasculares pulmonares están elevadas, por lo tanto existe poca diferencia de presión entre la aorta y la pulmonar, de modo que la velocidad de flujo transductal puede ser insuficiente para generar soplo u otros signos de persistencia del conducto arterioso. Definitivamente, el método más confiable para diagnosticar persistencia del conducto arterioso significativo es la ecocardiografía. ${ }^{5,6,10,19}$ Este análisis hizo que el criterio de selección de los pacientes para recibir tratamiento con paracetamol intravenoso se basará en los hallazgos ecocardiográficos y no en los clínicos.

Los pacientes recibieron tratamiento con paracetamol inmediatamente después del diagnóstico ecocardiográfico, en promedio a los seis días de vida. La literatura recomienda que en general se solicite ecocardiografía a los recién nacidos prematuros entre las 48 y 72 horas de vida, cuando teóricamente se ha producido el cierre funcional del conducto arterioso. ${ }^{16,17,19} \mathrm{El}$ diagnóstico ecocardiográfico, y por ende el inicio de tratamiento en estos pacientes se retrasó por circunstancias ajenas a los autores, ya que en todos fue solicitado al tercer día de vida.

En nuestro estudio la frecuencia de cierre del conducto arterioso fue de $89 \%$, similar a la obtenida en recién nacidos prematuros por Hammerman y por Yetka, ${ }^{16,17}$ quienes describen una frecuencia de cierre con paracetamol oral de $87.5 \%$ y $100 \%$, respectivamente. En estudios con paracetamol intravenoso efectuados por Oncel y por Serafettin ${ }^{20,21}$ el cierre del conducto arterioso fue de $100 \%$. En el único paciente en el que no se consiguió el cierre del conducto arterioso se administró previamente ibuprofeno oral por falta de disponibilidad temporal de paracetamol intravenoso y se decidió, después de tres días de tratamiento con este fármaco, la administración de paracetamol intravenoso ante la falta de cierre del conducto arterioso.

Nuestro estudio reafirma que el paracetamol puede ser una excelente alternativa en esta afección ya que su eficacia en el cierre del conducto arterioso es superior a la descrita en las publicaciones para el ibuprofeno y para la indometacina, que en promedio tienen una eficacia de cierre de 70 a $80 \% .{ }^{11,16,17}$ Una ventaja adicional es que el paracetamol intravenoso pudo administrarse en pacientes sin posibilidad 
de recibir medicamento por vía oral, como los que tenían enterocolitis necrosante o que no tenían un volumen significativo de la misma, lo que en nuestro medio no puede indicarse con el ibuprofeno ya que sólo se cuenta con la presentación oral.

El tiempo promedio de cierre del conducto arterioso después de la administración de paracetamol de $3.4 \pm 1.7$ días sugiere también que este fármaco es eficaz en el tratamiento de esta enfermedad y comparable con los días de tratamiento con indometacina e ibuprofeno que se requieren para el cierre del conducto arterioso. ${ }^{16,17}$

En este estudio no encontramos ninguna reacción adversa a la administración de paracetamol intravenoso, lo que puede constituir una ventaja adicional en comparación con los fármacos que actualmente se utilizan para el tratamiento de esta afección. Sin embargo, este estudio no fue específicamente diseñado para detectar eventos adversos.

Este es un estudio preliminar cuyas observaciones deben ser corroboradas por estudios prospectivos, aleatorizados y ciegos que comparen la eficacia del paracetamol con fármacos utilizados rutinariamente en el tratamiento de la persistencia del conducto arterioso, como la indometacina y el ibuprofeno, para obtener conclusiones definitivas. Sería importante, además, estudiar la eficacia y seguridad de este fármaco en prematuros con peso menor a 1000 gramos y la edad posnatal máxima en la que puede emplearse en el recién nacido.

\section{REFERENCIAS}

1. Noori S, McCoy M, Friedlich P, Bright B, Gottipati V, Seri I, et al. Failure of Ductus Arteriosus Closure Is Associated With Increased Mortality in Preterm Infants. Pediatrics 2009;123(1):e138-144.
2. Evans N, Malcolm G, Osborn D, Kluckow M. Diagnosis of Patent Ductus Arteriosus in Preterm Infants. Neo Reviews 2004;5:e86-97.

3. Alva EC. Lo esencial de la cardiología. 1ra ed. México: McGraw-Hill Interamericana. 2006;73-81

4. Bökenkamp-Gramann, R., 2012, Doctoral thesis, Leiden University "The ductus arteriosus: a fetal vessel coming of age." ISBN 9789461083333

5. Tapia-Rombo CA, Calvo-Rangel KM, Saucedo-Zavala VJ, Mora-Fol JR, Santiago-Romo JE. Evolución final de recién nacidos prematuros operados y no operados por persistencia del conducto arterioso. Gac Méd Méx 2008;144:111120.

6. Evans N, Malcolm G, Osborn D, Kluckow M. Diagnosis of Patent Ductus Arteriosus in Preterm Infants. Neo Reviews 2004;5:e86-97.

7. Clyman RI. Patent ductus arteriosus in preterm neonates. In: Avery's diseases of the newborn. Eds: Taeush HW, BaIlard RA. Philadelphia.7th ed. WB Saunders pp. 699-710.

8. Medrano LC, Guía TJM, Rueda NF, Moruno TA. Actualización en cardiología y cardiopatías congénitas. Rev Esp Cardiol 2009;62:39-52.

9. Skinner J. Diagnosis of patent ductus arteriousus. Sem Neonatol 2001;6:49-61.

10. Urquhart DS, Nicholl RM. How good is clinical examination at detecting a significant patent ductus arteriousus in the preterm neonate?. Arch Dis Child 2003;88:85-86.

11. Cherif A, Khrouf N, Jabnoun S, Mokrani C, Moez Ben A, Guellouze N, Kacem S. Randomized Pilot Study Comparing Oral Ibuprofen With Intravenous Ibuprofen in Very Low Birth Weight Infants With Patent Ductus Arteriosus. Pediatrics 2008;122:e1256-1261.

12. De Carolis MP, Romagnoli C, Polimeni V, Piersigilli F, Zecca $E$, Pappacci $P$ et al. Prophylactic ibuprofen therapy of patent ductus arteriosus in preterm infants. Eur J Pediatr 2000;159:364-368.

13. Petra M, Lemmers A, Toet MC, van Bel F. Impact of Patent Ductus Arteriosus and Subsequent Therapy with Indomethacin on Cerebral Oxygenation in Preterm Infants. Pediatrics 2008;121:142-147.

14. Zecca E, Romagnoli C, De Carolis M, Costa S, Marra R, De Luca D. Does Ibuprofen increase neonatal hyperbilirubinemia? Pediatrics 2009;124:480-484.

15. Quinn D, Cooper B, Clyman R. Factors associated with permanent closure of the Ductus Arteriosus: A Role for Prolonged Indomethacin Therapy. Pediatrics 2002;110; e10.

16. Hammerman C, Bin-Nun A, Markovitch E, Schimmel MS, Kaplan M, Fink D. Ductal closure with Paracetamol: A Surprising New Approach to Patent Ductus Arteriousus. Pediatrics 2011;128:e1618-1621.

17. Oncel MY, Yurttutan S, Uras N, Altug N, Ozdemir R, et.al "An alternative drug (paracetamol) in the management 
of patent ductus arteriosus in ibuprofen-resistant or contraindicated preterm infants". Arch Dis Child Fetal Neonatal Ed. 2013;98(1):F94 doi:10.1136/archdischild-2012-302044.

18. Bell EF, Acarregui MJ. Restricted versus liberal water intake for preventing morbidity and mortality in preterm infants. Cochrane Database Syst Rev 2000;(2):CD000503.

19. Golombek SG, Sola A, Baquero H, Borbonet D, Cabañas F, Fajardo C. Primer consenso clínico de SIBEN: enfoque diagnóstico y terapéutico del Ductus arterioso permea- ble en recién nacidos pretérmino. An Pediatr (Barc) 2008;69(5):454-81.

20. Oncel MY, Yurttutan S, Degirmecioglu H, Uras N, Altug N, Erdeve $\mathrm{O}$, et al. Intravenous Paracetamol Treatment in the Management of Patent Ductus Arteriousus in Extremely Low Birth Weight Infants. Neonatology 2013;103:166-169.

21. Serafettin TK, Ceviz N, Demirelli Y, Olgun H, Caner I, Oguz SI, et al. Intravenous Paracetamol for Patent Ductus Arteriousus In Premature Infants- A Lower Dose Is Also Effective. Neonatology 2013;104:6-7.

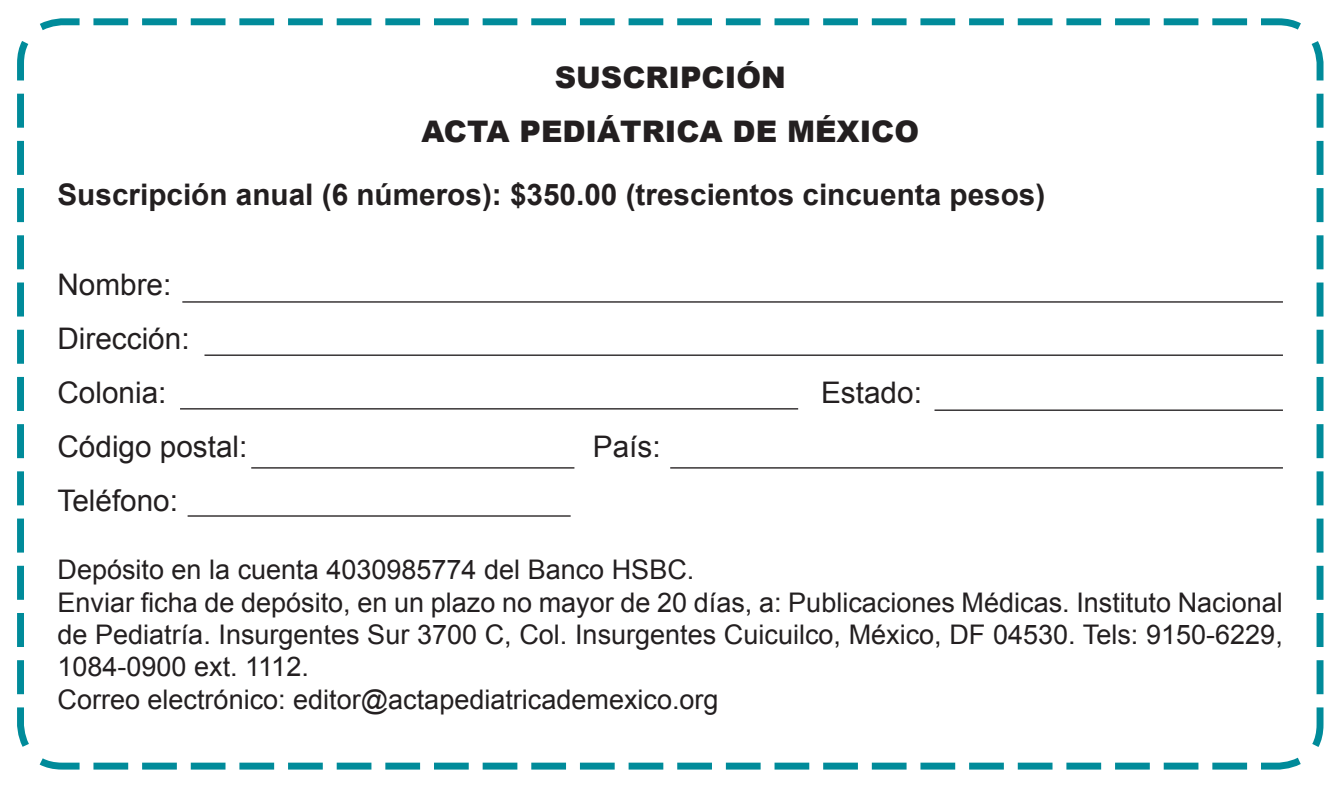

